\title{
Boundary-to-boundary flows in planar graphs
}

\author{
Glencora Borradaile $^{1}$ and Anna Harutyunyan ${ }^{2, \star}$ \\ 1 Oregon State University \\ 2 Vrije Universiteit Brussel
}

\begin{abstract}
We give an iterative algorithm for finding the maximum flow between a set of sources and sinks that lie on the boundary of a planar graph. Our algorithm uses only $O(n)$ queries to simple data structures, achieving an $O(n \log n)$ running time that we expect to be practical given the use of simple primitives. The only existing algorithm for this problem uses divide and conquer and, in order to achieve an $O(n \log n)$ running time, requires the use of the (complicated) linear-time shortest-paths algorithm for planar graphs.
\end{abstract}

Keywords: maximum flow, multiple terminal, planar graphs

\section{Introduction}

The problem of finding maximum flow in planar graphs has a long history, starting with the work of Ford and Fulkerson [7] in which the Max-flow, Min-cut Theorem was proved and the augmenting-paths algorithm was introduced. Since then, algorithms for maximum flow in planar graphs have fallen into one of three paradigms: augmenting paths, divide and conquer using small balanced planar separators, or via shortest paths in the dual. We note a subset of these results that are relevant to this paper. Borradaile and Klein gave an augmenting-paths algorithm for maximum st-flow in directed planar graphs that uses dynamic trees to achieve an $O(n \log n)$ running time [3]. For the special case when $s$ and $t$ are on the same face, an augmenting-paths algorithm can be simulated via Dijkstra's algorithm or, equivalently, determined from shortest-path distances in the dual graph 9 (details in Section 2). Borradaile et al. gave a rather complicated $O\left(n \log ^{3} n\right)$-time divide-and-conquer algorithm for when there are multiple sources and sinks (not necessarily on a common face) 4. For the special case when these sources and sinks are all on a common face 1 (such as the boundary of the embedded graph), Miller and Naor gave a simpler divide-and-conquer algorithm [12.

In this work we give an iterative algorithm for this last boundary-to-boundary case. While our algorithm does not improve on the asymptotic running time of Miller and Naor's work, in order for Miller and Naor's algorithm to be implemented in $O(n \log n)$ time, one requires repeated applications of the linear-time

\footnotetext{
^ Work done while at Oregon State University.

${ }^{1}$ Note that there is no planarity-maintaining reduction from this case to the singlesource, single-sink case.
} 
shortest-paths algorithm of Henzinger et al. [10. This shortest-paths algorithm is arguably impractical: it is also a divide-and-conquer algorithm using small planar separators, involves 'large constants' and, to our knowledge, has not been implemented. Our algorithm, on the other hand, requires just $O(n)$ (with a small constant) queries to simple data structures: namely a priority queue and a linked list 6].

Our algorithm is an augmenting-paths algorithm that iterates over the sourcesink pairs. We simulate finding the flow between a given source and sink using Hassin's method - via Dijkstra's algorithm in the dual graph. In order to prevent searching the same region of the graph multiple times, we search the graph in a biased way [8], such that we need only reuse the boundary of the searched region for augmenting further source-sink pairs. In order to reuse these boundaries efficiently, we use a simple generalization of priority queues in which queues are merged whose relative priorities differ by a constant or offset. These offset queues are implemented using edge weights to encode the offset in a tree implementation of the heap; doing so does not affect the asymptotic running time of the basic priority queue operations. Details are given in Appendix A.

We believe that the methods used in this paper may be applicable to other planar flow problems. For example, in a companion paper [2], we argue that the augmenting-paths algorithm of Borradaile and Klein for maximum st-flow in directed planar graphs can also be simulated by Dijkstra in the dual graph; the details of the implementation in this paper may lead to an $O(n \log n)$ algorithm for maximum st-flow in directed planar graphs that does not require the more cumbersome dynamic-trees data structure.

\section{$1.1 \quad$ Definitions}

We give a brief outline of definitions where we may stray from convention. For more complete and formal definitions, please refer to Borradaile's dissertation [5]. We extend any function or property on elements to sets of elements in the natural way.

Our algorithms are for directed graphs, but we consider the underlying undirected graph where each edge has two oppositely directed darts. Darts are oriented from tail to head. Capacities, $\boldsymbol{c}$, on the darts are positive and asymmetric, reflecting the original directed problem. Paths and cycles are sequences of darts and so are naturally directed; a path or a cycle may visit the same vertex multiple times; those that do not are simple; a path may be trivial, in which case it is a vertex. $X[a, b]$ denotes the $a$-to- $b$ subpath of $X$ where $X$ is a path, cycle or tree; $\circ$ denotes the concatenation of paths (which may result in a cycle).

A flow $\boldsymbol{f}$ is an assignment of real numbers to darts that is antisymmetric (for a dart and its reverse), respects capacities and is balanced at all non-terminal (non-source, sink) vertices. The value $|\boldsymbol{f}|$ of a flow is the net flow entering the sinks. A flow is a circulation if there are no terminals. The residual capacities $\boldsymbol{c}_{f}$ of capacities $\boldsymbol{c}$ w.r.t. flow $\boldsymbol{f}$ are given by:

$$
\boldsymbol{c}_{\boldsymbol{f}}[d]=\boldsymbol{c}[d]-\boldsymbol{f}[d], \forall \text { darts } d
$$


A path or cycle $X$ is residual if the residual capacity of every dart in $X$ is strictly positive. A dart is saturated if its residual capacity is zero. Residuality is w.r.t. capacities (such as $\boldsymbol{c}$ or $\boldsymbol{c}_{\boldsymbol{f}}$ ).

An $x y$-cut in $G$ is a set of darts $C$, the removal of which leaves no $x$-to- $y$ paths. The value of a cut is the total capacity of its darts. The value of the minimum $x y$-cut equals to that of the maximum $x y$-flow [7].

We use the usual definitions for planar graphs and their duals. We denote any path, cycle, vertex, face, dart in the dual graph with a $*$-superscript. If $d$ is a dart in $G$, then $d^{*}$ is the corresponding dual dart; if $v$ is a vertex and $f$ is a face in $G$, $v^{*}$ is a face and $f^{*}$ is a vertex in $G^{*}$. The boundary of the graph is denoted $\partial G$ and is taken to be clockwise. We refer to simple cycles as being clockwise (c.w.) or counterclockwise (c.c.w.); c.w. and c.c.w. depend on the choice of infinite face, $f_{\infty}$, which, throughout this paper, we will take to be the face common to all the sources and sinks.

For two non-crossing $x$-to- $y$ paths $P$ and $Q$, we say $P$ is left of $Q$ if $P \circ \operatorname{rev}(Q)$ is c.w. A path is leftmost if there are no paths left of it. For an $x$-to-y path $P$ that starts and ends on $\partial G$, we say a face, edge, path, etc. $X$ is (strictly) left of $P$ if $X$ is (strictly) contained by the c.w. cycle $\partial G[x, y] \circ \operatorname{rev}(P)$. We say that a planar flow $\boldsymbol{f}$ is leftmost if every c.w. cycle is non-residual w.r.t. $\boldsymbol{c}_{\boldsymbol{f}}$. We say that capacities are c.w. acyclic if every c.w. cycle is non-residual w.r.t. the capacities.

\section{Leftmost maximum flows and shortest paths}

Khuller, Naor and Klein [1] showed that a flow that is derived from shortestpath distances in the dual is c.w. acyclic. Formally:

Theorem 1 (Clockwise acyclic flows). Let $\boldsymbol{d}$ be the shortest-path distances in $G^{*}$ from $f_{\infty}^{*}$ interpreting capacities as lengths. Then every c.w. cycle is nonresidual w.r.t. the flow

$$
\boldsymbol{f}[d]=\boldsymbol{d}\left[\text { head }\left(d^{*}\right)\right]-\boldsymbol{d}\left[\operatorname{tail}\left(d^{*}\right)\right] \forall \text { darts } d
$$

where head $\left(d^{*}\right)$ and tail $\left(d^{*}\right)$ are the head and tail vertices of $d^{*}$ in $G^{*}$.

Earlier, Hassin had used this idea to find a maximum st-flow in an st-planar graph [9. We can view his algorithm by turning it into a circulation problem: introduce a new infinite-capacity arc $t s$ embedded so that every $s$-to- $t$ residual path forms a c.w. cycle with $t s$ and then saturate the c.w. cycles. We describe an equivalent formulation which we use in this paper. Split the dual vertex $f_{\infty}^{*}$ into two vertices $a_{\infty}^{*}$ and $b_{\infty}^{*}$ such that all the darts in $\partial G[s, t]^{*}$ are incident to $a_{\infty}^{*}$ and all the darts in $\partial G[t, s]^{*}$ are incident to $b_{\infty}^{*}$; denote the resulting graph $G_{s t}^{*}$. Let $\boldsymbol{d}\left[x^{*}\right]$ be the shortest-path distance from $a_{\infty}^{*}$ to $x^{*}$ in $G_{s t}^{*}$, viewing capacities as lengths. Then the flow assignment $\boldsymbol{f}_{s t}$ for $G$ given as in Equation (2) is a maximum $s t$-flow. It follows directly from Theorem 1 that $\boldsymbol{f}_{s t}$ is the leftmost maximum st-flow.

Since simple cuts in the primal map to simple cycles in the dual (and vice versa) [13, the darts of an $s t$-cut $C$ form an $a_{\infty}^{*}$-to- $b_{\infty}^{*}$ path $C^{*}$ in $G_{s t}^{*}$. If $C$ is a minimum cut, $C^{*}$ is a shortest path. 
Observation 1 A leftmost flow w.r.t. c.w. acyclic residual capacities is acyclic. [3]

Because of this acyclicity, one can easily show:

Observation 2 Let $\boldsymbol{c}$ be c.w. acyclic capacities and let $\boldsymbol{f}$ the leftmost, max st-flow for $s$ and $t$ on $f_{\infty}$. Then there is a decomposition of $\boldsymbol{f}$ into unique, noncrossing s-to-t paths $P_{1}, P_{2}, \ldots, P_{\ell}$ where $P_{i}$ carries $f_{i}>0$ units of flow and $P_{i}$ is left of $P_{j} \forall i<j$. Further, an augmenting-paths algorithm that always saturates the leftmost path first saturates the paths $P_{1}, \ldots, P_{\ell}$ in order.

Our algorithm requires c.w. acyclic capacities; the analysis will use this fact indirectly by invoking Observation 2. We will achieve this property in a preprocessing step and maintain this as an invariant throughout the algorithm. It follows from Equation (2) and Observation 2 that, for every primal face $x$ (dual vertex $\left.x^{*}\right)$ :

$$
\boldsymbol{d}\left[x^{*}\right]= \begin{cases}\sum_{j=1}^{i} f_{j} & \text { if } x \text { is right of } P_{i} \text { and left of } P_{i+1} \\ \sum_{j=1}^{\ell} f_{j}=|\boldsymbol{f}| & \text { if } x \text { is right of } P_{\ell}\end{cases}
$$

\section{1 st-planar flow via biased search}

We describe how to find an st-planar flow via biased search (in the dual) that does not necessarily search the entire graph, assuming that the initial capacities are c.w. acyclic. We assume that there are no degree- 2 vertices in the primal; any such vertex could be removed by merging the adjacent darts (in each direction) and keeping the minimum of the capacities. Parallel darts (not antiparallel) can be merged by taking the sum of their capacities. We additionally assume that the finite faces of the primal are triangulated (which can be achieved by the addition of 0-capacity edges).

We implicitly and iteratively build a decomposition as given in Observation 2 using Dijkstra's algorithm in the dual. Initially $P_{1}=\partial G[s, t]$. In phase $i$, we have already found path $P_{i}$; we maintain that, at the start of phase $i$, the faces adjacent to and right of $P_{i}$ are in the queue $Q_{i}$. (Keep in mind that faces are vertices in the dual, and we are really just finding shortest paths in the dual graph, applying the standard rules for Dijkstra's algorithm.) The priority of face $x$ is the capacity of the minimumcapacity dart bounding $x$ in $P_{i}$. Say the minimum priority in the queue is $q$; to find $P_{i+1}$ we pop faces off the

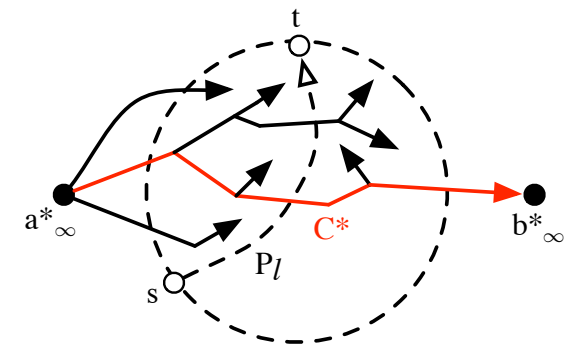

Fig. 1. $\partial G$ is the dashed circle and the dashed $s$-to- $t$ path is $P_{\ell}$. In $G_{s t}^{*}, a_{\infty}^{*}$ is incident to the duals of all the arcs on the path of the circle c.w. from $s$ to $t$ and $b_{\infty}^{*}$ is incident to the duals of all the arcs on the path of the circle c.w. from $t$ to $s$. The solid tree is the search tree used in the biased search algorithm with the $a_{\infty}^{*}$-to- $b_{\infty}^{*}$ path representing the leftmost cut $C^{*}$. 
queue with priority $q$ until the minimum priority in the queue is $>q$. Now we have popped off all the faces between $P_{i}$ and $P_{i+1}$ (by Equation (3)) and $Q_{i+1}$ contains all the faces to the right of and adjacent to $P_{i+1}$.

So far, we have just described Hassin's algorithm, but have made explicit the augmenting paths that are implicit in his algorithm. We have also identified phases. In each phase, all the faces of a given distance label are explored via 0 -length darts (in the dual).

We modify the algorithm so that we do not explore the entire graph. Note that all the faces to the right of $P_{\ell}$ (the last augmenting path), by Equation (3) have distance label $|\boldsymbol{f}|$. Rather than label all these faces, after getting to the start of phase $\ell$, we wish to find the leftmost cut. Let $C^{*}$ be the leftmost, shortest $a_{\infty^{*}}^{*}$ to- $b_{\infty}^{*}$ path in $G_{s t}^{*}$; $C$ is the leftmost cut. The part of $C^{*}$ that is strictly to the right of $P_{\ell}$ consists of 0-length darts, since the sum of the capacities of the darts in $C^{*}$ that are in $P_{1}, \ldots, P_{\ell}$ is $|\boldsymbol{f}|$ by Equation (3). In addition to identifying the leftmost cut, we wish to not explore any part of the graph strictly right of $P_{\ell}$ and $C^{*}$. (See Figure 1.)

We find the leftmost cut by at each phase additionally maintaining an ordering $A_{i}$ of the faces in $Q_{i}$ that reflects their order along $P_{i}$ from $t$ to $s$. We maintain and query the ordering using the order maintenance data structure DSORDER due to Dietz and Sleator [6] which is a circularly linked list with order information determined using 2's complement arithmetic. (See Appendix B for details. Each of the operations takes either $O(1)$ or $O(\log n)$ time per visited face.) During a phase, we:

(1) Start with faces that are closest to $t$ in the ordering.

(2) Explore along 0-length darts in the dual in a depth-first leftmost fashion; this can be done by following the combinatorial embedding of the darts around a vertex in a c.w. order, using the parent dart in the search tree implicit to Dijkstra's algorithm [3].

(3) If we reach $b_{\infty}^{*}$ during this search, we immediately stop the algorithm. (More details of this are given below.)

(4) At the end of this 0-length exploration, we remove from the queue and order any faces that we have reached in this exploration. Suppose $T^{*}$ is the dual search tree we have explored that contains the shortest paths found by Dijkstra's algorithm, rooted at a face adjacent to $P_{i}$. We add the never-visited faces adjacent to $T^{*}$ in their c.w. order around $T^{*}$ (according to their shortest adjacency to $T^{*}$ ). This ordering is easily visualized by contracting the edges of $T^{*}$ and considering the c.w. ordering of the darts around the new (dual) vertex.

At the start of each phase, the queue and the order contain the same set of elements. The leftmost-bias to the search additionally guarantees that the final dual search tree $T^{*}$ contains leftmost shortest paths. This can be easily shown via induction. Since we stop as soon as we reach $b_{\infty}^{*}$ and we search in a leftmost fashion, $T^{*}$ does not contain any darts strictly right of both the last flow path $P_{\ell}$ found and $T^{*}\left[a_{\infty}^{*}, b_{\infty}^{*}\right]$. In this way, we also guarantee:

Observation 3 At the end of this biased search, the queue and order contain the faces adjacent to and right of $P_{\ell}$. 
In our multi-source, multi-sink algorithm, we will reuse this queue and order. To do so, we need to know the residual capacities of the darts in $P_{\ell}$. If a face $f$ in the queue has exactly one bounding arc in $P_{\ell}$, then the priority of $f$ reflects exactly the residual capacity of that dart. If $f$ has two bounding darts $d_{1}$ and $d_{2}$ in $P_{\ell}$ (i.e., the head of $d_{1}^{*}$ and $d_{2}^{*}$ in $G^{*}$ is $f^{*}$ ), then, to the right of $P_{\ell}$, we can only push the minimum of these darts' residual capacities along this section of $P_{\ell}$. (Put another way, if we remove everything strictly to the left of $P_{\ell}, d_{1}$ and $d_{2}$ would be incident to a degree 2 vertex, which we would remove according to the rule at the start of this section.) We get:

Observation 4 The priority of a face $f$ in the queue reflects the residual capacity of the dart(s) bounding the face in $P_{\ell}$; the residual capacity is the priority less $|\boldsymbol{f}|$.

Subtracting $|\boldsymbol{f}|$ from the priorities in the ending queue can be done in $O(1)$ time using offset queues (Appendix A). Finally, the DSORDER data structure does not allow us to pull the first element of the order (having minimum priority in the queue) but does allow us to sort a subset of items. In doing so, we spend $O(\log n)$-amortized time per element. We do not wish to repeat this work. If we reach $b_{\infty}^{*}$ in the middle of a phase and have a subset of items $X$ that we have sorted using DSORDER, we break the ties in the priorities of these items in the priority queue. When we return to use this queue/order, we will not need to resort these items.

\section{Algorithm}

For simplicity of presentation we will assume that the terminals are alternating sources and sinks along $\partial G$. This can be attained by taking a consecutive group of sources $S$, introducing a new source and connecting the new source to every source in $S$ with an infinite capacity arc. We number the sources and sinks according to their c.w. ordering on $\partial G, s_{1}, t_{1}, s_{2}, t_{2}, \ldots, s_{m}, t_{m}$, starting with an arbitrary source. We return the difference between the original capacities and final residual capacities, which, by Equation (1), is the corresponding flow.

AbstractFlow $\left(G,\left\{s_{1}, t_{1}, s_{2}, t_{2}, \ldots, s_{m}, t_{m}\right\}, \boldsymbol{c}\right)$

Saturate all $s_{j}$-to- $t_{i}$ residual paths $\forall i<j$ and all c.w. cycles.

Let $\boldsymbol{c}_{0}$ be the resulting residual capacities.

For $j=1,2, \ldots, m$ :

for $i=j, j-1, \ldots, 1$ :

let $\boldsymbol{c}_{i j}^{\prime}$ be the current residual capacities.

Find the leftmost $s_{i}$-to- $t_{j}$ flow $\boldsymbol{f}_{i j}$ w.r.t. $\boldsymbol{c}_{i j}^{\prime}$.

Let $\boldsymbol{c}_{i j}$ be the residual capacities of $\boldsymbol{c}_{i j}^{\prime}$ w.r.t. $\boldsymbol{f}_{i j}$.

Return $\boldsymbol{c}[d]-\boldsymbol{c}_{m m}[d]$ for all darts $d$.

The first step can be done with one shortest-path computation in the dual as follows (in $O(n \log n)$ time using Dijkstra's algorithm, for example); refer to 
(a)

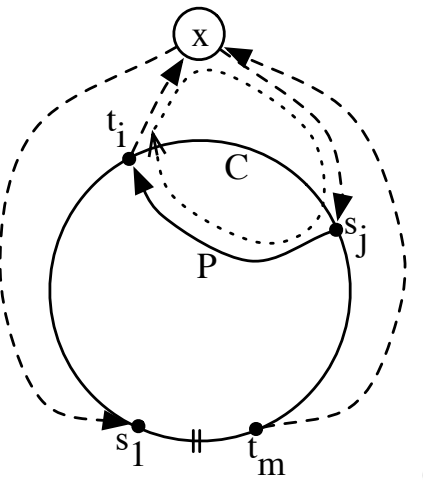

(b)

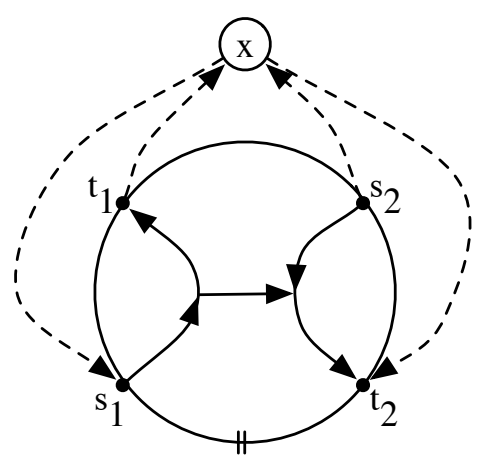

Fig. 2. (a) Illustrating the first step of the AbstractFlow. (b) A simple example illustrating why this first step cannot be repeated to find the overall maximum flow. The equivalent step would saturate all c.c.w. cycles. If the solid edges have equal capacity, this would saturate the $s_{1}$-to- $t_{2}$ path, since the method for saturating all c.c.w. cycles (like for c.w. cycles) saturates all largest such cycles. However, doing so would create a residual path from $s_{2}$ to $t_{1}$.

Figure 2(a). Embed a vertex $x$ in $f_{\infty}$. Connect $x$ to every source and every sink with infinite-capacity arcs. Embed these arcs so that $s_{1}, t_{m}$ and $x$ are on the infinite face. Let $\boldsymbol{f}$ be the circulation that saturates all the c.w. residual cycles in this graph (Theorem 1). Let $\boldsymbol{c}_{0}$ be the residual capacities of the darts in $G$ w.r.t. $\boldsymbol{f}$. Consider any simple path $P$ from $s_{j}$ to $t_{i}$ in $G$. For $j>i, P \circ t_{i} x \circ x s_{j}$ is a c.w. cycle $C$. Therefore $C$ must be non-residual w.r.t. $\boldsymbol{c}_{0}$ and, since the arcs $t_{i} x$ and $x s_{j}$ have infinite capacity, $P$ must be non-residual w.r.t. $\boldsymbol{c}_{0}$.

Note that while the iterative part of the algorithm saturates all $s_{i}$-to- $t_{j}$ paths $\forall i<j$, we cannot achieve this with a symmetric application of the first step. The simple example in Figure 2(b) illustrates why.

In the remainder of the paper we will give an efficient implementation of the double loop of ABSTRACTFLOW. We first show that the abstract algorithm guarantees several useful invariants that limit the region of the graph that is involved in each iteration. These invariants allow us to explore the graph in such a way that no region is explored multiple times. Correctness of ABSTRACTFLOW will also follow from these invariants. By iteration $i, j$, we will mean iteration $i$ of the inner loop and iteration $j$ of the outer loop.

\section{$3.1 \quad$ Invariants}

Since only leftmost flows are augmented we get (by definition and induction):

Invariant 1 There are no clockwise residual cycles in $G$ w.r.t. $\boldsymbol{c}_{i j}, \forall i \leq j$.

Since the sink is in common to all the iterations of the inner loop, for a given iteration of the outer loop, we get: 
Invariant 2 There are no residual $s_{j}$-to- $t_{k}$ paths w.r.t. $\boldsymbol{c}_{i, k}$ for $j>i$.

More formally, this follows from the Sinks Lemma [4. The following invariant shows that we do not undo the progress made by the first step of ABSTRACTFLOW.

Invariant 3 There are no $s_{i}$-to-t $t_{j}$ residual paths s.t. $i>j$ w.r.t. $\boldsymbol{c}_{0}$ or $\boldsymbol{c}_{k \ell}$, $\forall k<\ell$.

Proof. We prove this invariant by induction. It holds w.r.t. $\boldsymbol{c}_{0}$ as argued in Section 2. For a contradiction, let $\boldsymbol{c}_{k \ell}$ be the first residual capacities that introduce an $s_{i}$-to- $t_{j}$ residual path $R(i<j)$. Then there must be an $s_{k}$-to- $t_{\ell}$ path $A$ that is augmented in iteration $k, \ell$ and that uses a dart $d$ in $\operatorname{rev}(R)$.

Let $x$ and $y$ be the last and first, resp., vertices of $R$ that are in $A$. $A$, $R\left[s_{i}, y\right]$ and $R\left[x, t_{j}\right]$ are residual w.r.t. $\boldsymbol{c}_{k \ell}^{\prime}$ (the residual capacities at the start of iteration $k, \ell$ ). It follows that $k \leq j$ and $\ell>i$, for otherwise we contradict the inductive hypothesis. However, iteration $k, \ell$ comes after $i, \ell$ in ABSTRACTFlow. Invariant 2 tells us that there cannot be an $s_{i}$-to- $t_{\ell}$ path that is residual w.r.t. $c_{k \ell}^{\prime}$, contradicting the existence of $R\left[s_{i}, y\right] \circ A\left[y, t_{\ell}\right]$.

The optimality of the flow found by ABstractFlow follows from the last invariant (along with Invariants 2 and 3 :

Invariant 4 There are no $s_{i}$-to-t $t_{j}$ residual paths w.r.t. $\boldsymbol{c}_{\ell k}$ for any $\ell$ and any $k>j$.

Proof. We prove this invariant by induction. It holds w.r.t. $\boldsymbol{c}_{1, j+1}^{\prime}$ by Invariant 2 . For a contradiction, let $\boldsymbol{c}_{\ell k}$ be the first residual capacities that introduce an $s_{i^{-}}$ to- $t_{j}$ residual path $R$. W.l.o.g. assume that $i \leq j$ as the case $i>j$ is handled by Invariant 3 Then there must be an $s_{\ell}$-to- $t_{k}$ path $A$ that is augmented in iteration $\ell, k$ and that uses a dart $d$ in $\operatorname{rev}(R)$.

Let $x$ and $y$ be the first and last, resp., vertices of $R$ that $A$ shares. Since $A$ and $R\left[y, t_{j}\right]$ are residual, $\ell \leq j$ by Invariant 3 However, by Invariant 2 , there are no $s_{\ell}$-to- $t_{j}$ paths that are residual w.r.t. $\boldsymbol{c}_{1 j}$, so $\ell>j$, a contradiction.

\subsection{Unusability Structures}

We will illustrate our implementation of ABSTRACTFLOW with a recursive algorithm. To that end, we show that the cut and the flow found in iteration $i, j$ separates the graph into two pieces that act independently for the remainder of the algorithm. Let $P$ be the rightmost path in the path decomposition of $\boldsymbol{f}_{i j}$ given in Observation 2 (that has non-zero flow). The following lemma allows us to delete everything strictly to the left of $P$ at the end of iteration $i, j$ for future iterations without affecting optimality.

Lemma 1. There are no paths from $s_{k}$ to $P$ that are residual w.r.t. $\boldsymbol{c}_{i j}$ for $k>i$. 
Proof. First we make an observation. Inner iterations $j, j-1, \ldots, i$ are equivalent to adding a new source $s$, connecting $s$ to $s_{j}, s_{j-1}, \ldots, s_{i}$ by high-capacity arcs and saturating the leftmost max $s t_{k}$-flow ${ }^{2}$ By Observation 2, this is done by saturating a set of non-crossing $s$-to- $t_{k}$ paths $\mathcal{P}=P_{1}, P_{2}, \ldots$ ordered from left to right. In ABSTRACTFLOW, iteration $\ell, k$ will saturate a contiguous subset $\mathcal{P}_{\ell}$ of $\mathcal{P}$ for $i \leq \ell \leq j$. By saturating these paths in order, we first cut $s_{j}$ from $t_{k}$ by saturating $\mathcal{P}_{j}$, then cut $s_{j+1}$ from $t_{k}$ and so on.

For $i<k \leq j$, the lemma follows from the fact that iteration $k, j$ precedes $i, j$ : a path $Q$, from $s_{k}$-to- $P$ concatenated with the suffix of $P$, would be saturated before $P$. For $k>j, Q$ would be residual w.r.t. capacities $\boldsymbol{c}_{i j}^{\prime}$ since $\boldsymbol{f}_{i j}$ does not change the capacities of darts strictly to the right of $P ; Q$ violates Invariant 3 .

Let $C$ be the leftmost minimum $s_{i} t_{j}$-cut. The next lemma shows that we can delete the darts in $C$ (among others on the $t_{j}$ side of the cut) without affecting optimality. In the biased-search algorithm (Section 2.1), the darts satisfying Lemma 2 are exactly those that are searched to the right of the last flow path $\left(T^{*}\right)$ in finding the leftmost cut $(C)$.

Lemma 2. Let $W^{*}$ be any from-a $a_{\infty}^{*}\left|\boldsymbol{f}_{i j}\right|$-length path in $G_{s_{i} t_{j}}^{*}$ that is left of $C^{*}$. Then no s-to-t path that is residual w.r.t. $\boldsymbol{c}_{i j}$ uses a dart in $W$.

Proof. For a contradiction, suppose there is a $s_{k}$-to- $t_{\ell}$ path $R$ that is residual w.r.t. $\boldsymbol{c}_{i j}$ that uses a dart of $W$. Since, by Invariant $3, \ell \geq k, s_{k}$ must be on the $t_{j}$ side of $C$ for otherwise, $R$ would have to cross back and forth across $C$, but the darts of $C$ are only residual w.r.t. $\boldsymbol{c}_{i j}$ from the $t_{j}$ side to the $s_{i}$ side.

We have just finished iteration $i, j, k>j$, and so, by Invariant 3, there is an $s_{k} t_{j}$-cut $K$. Take $K$ to be the rightmost of these cuts (defined analogously to leftmost). In $G_{s_{i} t_{j}}^{*}, K^{*}$ is a c.c.w. cycle through $b_{\infty}^{*} ; K^{*}$ is 0-length (or, equivalently, composed entirely of darts that are non-residual w.r.t. $\boldsymbol{c}_{i j}$ ).

$K^{*}$ must be left of $C^{*}$, for otherwise, the leftmost-ness of $C^{*}$ and the rightmostness of $K^{*}$ would be violated. If $R$ uses a dart $d$ of $W$, then $d$ must be on the $s_{k}$ side of $K$. Then, in the dual, $W^{*}$ must intersect $K^{*}$ at a dual vertex $x^{*}$. But then $W^{*}\left[a_{\infty}^{*}, x^{*}\right] \circ K^{*}\left[x^{*}, b_{\infty}^{*}\right]$ is a $a_{\infty}^{*}$-to- $b_{\infty}^{*}$ path of length at most that of $W^{*}$; $W^{*}\left[a_{\infty}^{*}, x^{*}\right] \circ K^{*}\left[x^{*}, b_{\infty}^{*}\right]$ is left of $C^{*}$, contradicting that $C$ is a leftmost cut.

Lemmas 1 and 2 allow us to implement ABSTRACTFLow recursively. That is, AbstractRecursiveFlow, below, finds the same (non-zero) flows $\boldsymbol{f}_{i j}$ in the same order as ABSTRACTFLOW. The recursive algorithm has a slightly different input, as there may be several consecutive sources for the recursive calls. We illustrate the algorithm without explicitly returning the flow. It is trivial to determine the flow from the residual capacities found throughout the algorithm.

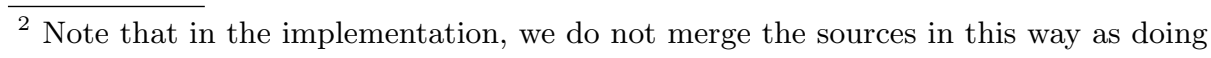
so does not allow us to reuse the work done in previous iterations.
} 
AbstractRecursiveFlow $\left(G,\left\{s_{1}, t_{1}, \ldots, s_{m}, t_{m}\right\}, \boldsymbol{c}\right)$

Saturate all $s_{j}$-to- $t_{i}$ residual paths $\forall i<j$ and all c.w. cycles.

Let $\boldsymbol{c}_{0}$ be the resulting residual capacities.

AbstractRecursiveFlowHelper $\left(G,\{\},\left\{s_{1}, t_{1}, \ldots, s_{m}, t_{m}\right\}, \boldsymbol{c}_{0}\right)$

AbstractRecursiveFlowHelper $\left(G,\left\{s_{1}, s_{2}, \ldots, s_{\ell-1}\right\},\left\{s_{\ell}, t_{\ell}, s_{\ell+1}, t_{\ell+1}, \ldots, s_{m}, t_{m}\right\}, \boldsymbol{c}\right)$

Find the leftmost $s_{\ell}$-to- $t_{\ell}$ flow $\boldsymbol{f}$ w.r.t. $\boldsymbol{c}$.

Let $\boldsymbol{c}^{\prime}$ be the residual capacities of $\boldsymbol{c}$ w.r.t. $\boldsymbol{f}$.

Let $P$ be the rightmost path in the path-decomposition of $f$ and let $C$ be the leftmost cut.

Let $G_{1}$ and $G_{2}$ be the components resulting from deleting all the darts strictly to the left of $P$ and the darts of $C$ from $G$.

If $t_{\ell} \in G_{2}$ :

Let $k$ be the greatest index s.t. $t_{k} \in G_{2}$.

AbstractRecursiveFlowHelper $\left(G_{2},\{\},\left\{s_{\ell+1}, t_{\ell+1}, \ldots, s_{k}, t_{k}\right\}, \boldsymbol{c}^{\prime}\right)$

Let $h$ be the smallest index $>\ell$ s.t. $t_{h} \in G_{1}$.

Extend $Q$ and $A$ to contain all the faces in $G_{1, s_{\ell} t_{h}}^{*}$ that are incident to $a_{\infty}^{*}$

AbstractRecursiveFlowHelper $\left(G_{1},\left\{s_{1}, s_{2}, \ldots, s_{\ell}\right\},\left\{s_{h}, t_{h}, \ldots, s_{m}, t_{m}\right\}, \boldsymbol{c}^{\prime}\right)$ Else:

Let $j$ be the greatest index $<\ell$ s.t. $s_{j} \in G_{1}$.

AbstractRecursiveFlowHelper $\left(G_{1},\left\{s_{1}, s_{2}, \ldots, s_{j}\right\},\left\{s_{\ell+1}, t_{\ell+1}, \ldots, s_{m}, t_{m}\right\}, \boldsymbol{c}^{\prime}\right)$

Lemma 3. AbstractRecursiveFlow implements AbstractFlow.

Proof. Refer to Figure 3. By Lemmas 1 and 2 the deleted edges are safe to remove: solving the problem in the two subproblems will indeed find an optimal solution. The $s_{\ell} t_{\ell}$ augmentation performed by ABSTRACTRECuRsiveFLOWHELPER corresponds to an iteration of ABSTRACTFLOW. If there are residual source-to- $t_{\ell}$ paths remaining after this augmentation, then there would necessarily be one such path from $s_{1}$, and $t_{\ell} \notin G_{2}$. AbstractRecursiveFLOWHELPER would continue to push flow from earlier sources to $t_{\ell}$, just as ABStractFlow. Otherwise, both Abstract- and AbstractRecursive-Flow would move onto the next sink, in which case $t_{\ell} \in G_{2}$.

\subsection{Reusing queues for an efficient implementation}

We show how to implement AbstractRecursiveFlow using $O(n)$ queries to simple data structures: the priority queue and DSORDER data structure (which is at heart a linked list). The challenge in doing so can be illustrated by a simple example. Suppose $s_{1}$ has a high-capacity path $P$ with many edges ending with a low-capacity star that connects to each of the sinks. In each iteration
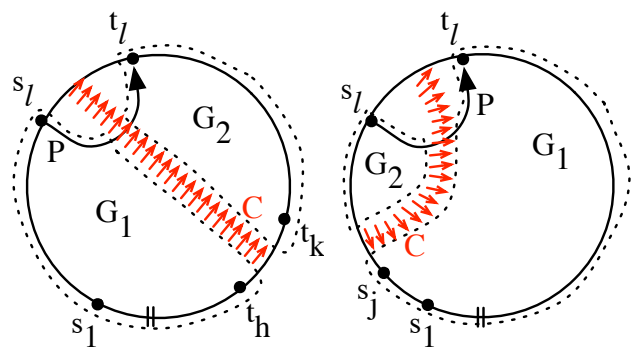

Fig. 3. The two cases for subproblems for $(\mathrm{AB}-$ Stract)RecursiveFlow. If $t_{\ell} \in G_{2}$ (left), there are 2 non-trivial subproblems. 
of the outer loop, we could require augmenting the flow along this long path. We overcome this barrier by reusing the work from earlier iterations in later iterations.

We give an implementation (RECURsiveFlow) of ABstractReCURsiveFlowHelper. To implement the first step of ABstractReCURSIVEFlowHelper, we use the biased-search algorithm described in Section 2.1. Note that the subproblem corresponding to terminal sets $\left\{s_{1}, s_{2}, \ldots, s_{\ell-1}\right\},\left\{s_{\ell}, t_{\ell}, s_{\ell+1}, t_{\ell+1}, \ldots, s_{m}, t_{m}\right\}$ results from having found maximum flows from $s_{1}, s_{2}, \ldots, s_{\ell-1}$ to $t_{\ell}$. We keep the queue and order at the end of the biased-search algorithm used to find these flows.

Formally, we will pass to RECURSIVEFLOw a queue and order for each source $s_{i}, i \leq \ell$. The queue $Q_{i}$ and order $A_{i}$ contains all the faces adjacent to and right of $\partial G\left[s_{i}, s_{i+1}\right]$ for $i<\ell$ and $\partial G\left[s_{i}, t_{i}\right]$ for $i=\ell$. The order reflects the c.c.w. ordering of the faces along $\partial G$. The priority of a face $f$ in $Q_{i}$ is the current residual capacity of the primal copy of the dart $f_{\infty}^{*} f^{*}$. Recall from Section 2.1 that the biased-search algorithm guarantees this at the end of the search.

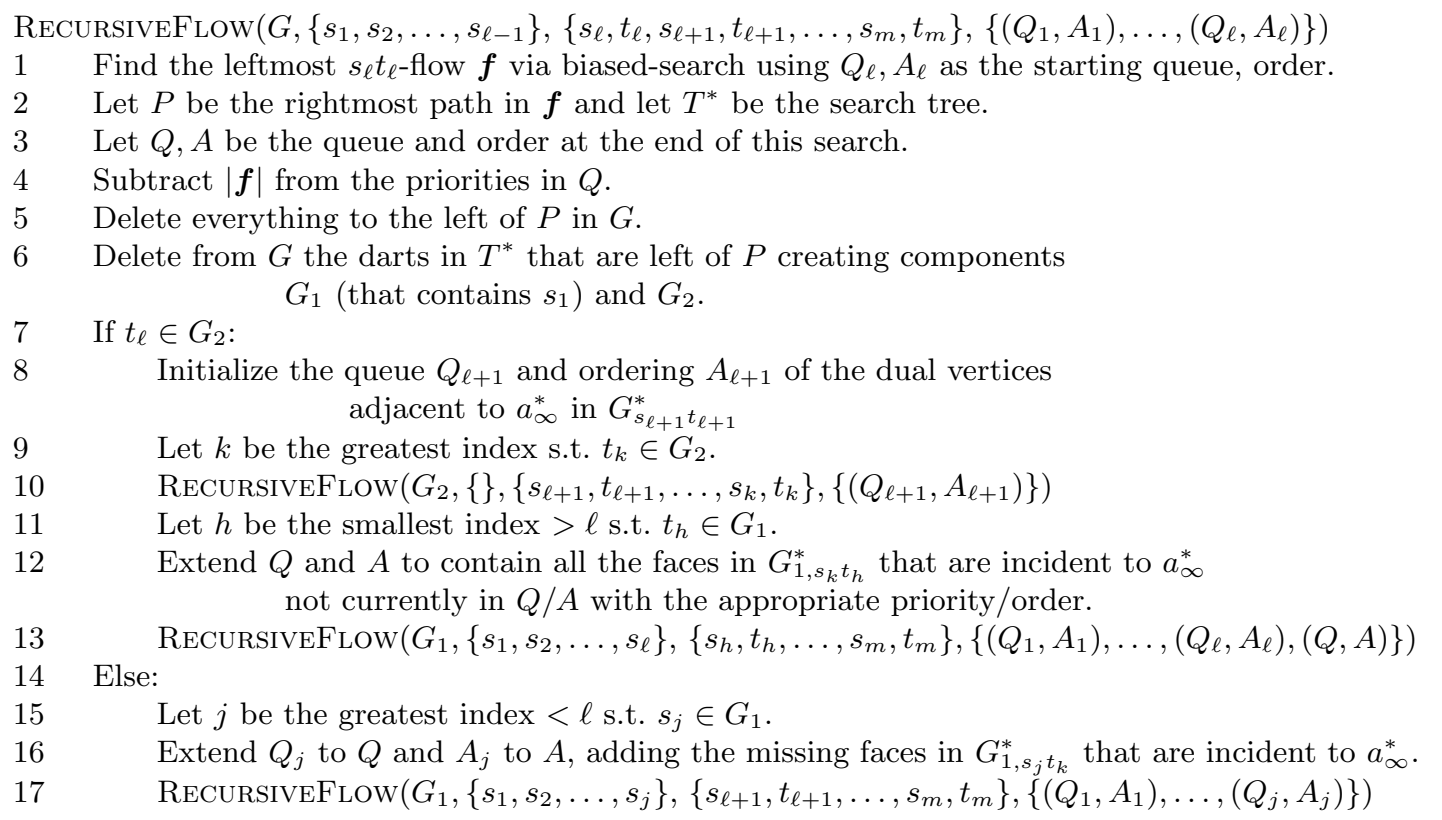

Running time and correctness of ReCuRsiveFLow By Observation 4. Step 4 results in the priorities reflecting exactly the residual capacities of the darts in $P$ after saturating $f . G_{1}$ and $G_{2}$ are the same as the subgraphs created in AbstractRecursiveFlow, as are the subproblems considered. The removed darts create a new boundary and so maintain triangulation of the finite faces. Step 12 can be done in $O(\log n)$ per new face added (Appendices $\mathrm{A}$ and B). Adding the faces can be achieved by a left-first search from $Q$ (or from $Q_{j}$ to $Q$ ); this creates the queue and order along the boundary of the graph. In order to combine the orders $A_{j}$ and $A$ in line 16, we observe that the order 
$A_{j}$ is guaranteed to be right of the order $A$ when they are joined together. The DSORDER data structure allows us to concatenate these orders efficiently (details in Appendix B).

Finally, we argue that the entire algorithm requires only $O(n)$ queries to priority queue and DSORDER data structure. The biased-search algorithm uses $O(k)$ priority-queue and DSORDER queries where $k$ is the size of the search tree discovered (Section 2.1). This is in part due to the triangulation of the finite faces; the degree of the vertices from which we search during the biased-search algorithm have degree 3 , so the 0-length darts leaving a vertex can be determined in constant time.

For the subproblem $G_{1}$, we start with queues that have already been initialized, so, as argued at the end of Section 2.1. we essentially pick up the search where we left off, not repeating any computation at the boundary where we left off (the rightmost path in a previous flow). For the subproblem $G_{2}, P$ forms part of the boundary and so part of the queue/order ending at $t_{\ell}$ appear in this subgraph. However, by Lemma 1, no residual path intersects $P$. Since the finite faces are triangulated, no path can intersect a face adjacent to $P$ without intersecting $P$. Therefore, none of the faces in the queue/order along $P$ will be used in the subproblem corresponding to $G_{2}$. It follows that there are a constant number of data-structure queries per finite face of the original graph.

\section{References}

1. M. Bender, R. Cole, E. Demaine, M. Farach-Colton, J. Zito. Two Simplified Algorithms for Maintaining Order in a List. In Proc. ESA, pages 152-164, 2002.

2. G. Borradaile and A. Harutyunyan. Maximum st-flow in directed planar graphs via shortest paths. To appear in Proc. IWOCA, 2013.

3. G. Borradaile and P. Klein. An $O(n \log n)$ algorithm for maximum st-flow in a directed planar graph. J. of the ACM, 56(2):1-30, 2009.

4. G. Borradaile, P. Klein, S. Mozes, Y. Nussbaum, C. Wulff-Nilsen. Multiple-Source Multiple-Sink Maximum Flow in Directed Planar Graphs in Near-Linear Time. In Proc. FOCS, pages 170-179, 2011.

5. G. Borradaile. Exploiting Planarity for Network Flow and Connectivity Problems. $\mathrm{PhD}$ thesis, Brown University, 2008.

6. P. Dietz and D. Sleator. Two algorithms for maintaining order in a list. In Proc. STOC, pages 365-372, 1987.

7. C. Ford and D. Fulkerson. Maximal flow through a network. Canadian J. Math., 8:399-404, 1956.

8. A. Goldberg and C. Harrelson. Computing the shortest path: A search meets graph theory. In Proc. SODA, pages 156-165, 2005.

9. R. Hassin. Maximum flow in $(s, t)$ planar networks. IPL, 13:107, 1981.

10. M. Henzinger, P. Klein, S. Rao, S. Subramanian. Faster shortest-path algorithms for planar graphs. JCSS, 55(1):3-23, 1997.

11. S. Khuller, J. Naor, P. Klein. The lattice structure of flow in planar graphs. SIAM J. on Disc. Math., 6(3):477-490, 1993.

Acknowledgements: This material is based upon work supported by the National Science Foundation under Grant No. CCF-0963921. 
12. G. Miller and J. Naor Flow in planar graphs with multiple sources and sinks. SIAM J. on Comp., 24(5):1002-1017, 1995.

13. H. Whitney Planar Graphs. Fundamenta Mathematicae, 21:73-84, 1933.

\section{A Priority queues with offsets}

We show how to efficiently change all the priorities in a queue by a fixed amount. This will be used when we wish to merge two priority queues whose relative priorities differ by a constant. That is, we have two priority queues $P$ and $Q$ that we want to merge, but the priorities of the items in $P$ are offset from those in $Q$ by some amount $o$. We illustrate this for a binomial-heap implementation of priority queues, but this technique is not limited to a specific implementation (although the details of handling the offsets will depend on the implementation).

For the purposes of this discussion the details of a binomial heap, beyond the fact that it is a set of rooted trees, are irrelevant. We refer the reader to any data structures textbook for details. We will argue that the standard operations (insert, find minimum, delete minimum, decrease key and merge) will have the same asymptotic running time with offsets as without. To do so, we annotate the edges of the trees in the heap with weights, initially zero. We give the roots of the trees a dummy parent edge so that every item in the queue (node in a tree) $x$ has a unique parental edge weight $w(x)$. We say that node $x$ has a local priority $p_{\ell}(x)$ and a global priority $p(x)$ where $p(x)$ is the sum of $p_{\ell}(x)$ plus the parental edge weights on the path to the root of the binomial tree containing $x$ (including the weight of the dummy root edge). Initially the global priorities are the same as the local priorities. We will maintain that the heap property holds for the global priorities (ie. my children's global priorities are lower than mine).

We describe the modifications we make to the binomial-heap-based priority queue operations:

insert Unchanged as insert reduces to merge.

find min The minimum priority element is guaranteed to be a root of one of the trees. When comparing the roots of the trees, first sum the local priority and dummy root edge weight.

delete min The standard operation is to delete the root that is the minimum priority element and then merge the resulting child trees with the remaining trees. We first add the weight of the dummy root edge to the weights of the child edges; these child edges become dummy parent edges of the trees before they are merged.

decrease key The standard operation traverses the path from the node in question, $x$ to the root and swaps nodes that violate the heap property. First compute the global priorities of the nodes on the $x$ to root path. Then traverse to the to-root path: say $x$ is a child of $y$ such that $p(x)<p(y)$; let $w$ be the weight of the edge $x y$. Swap $x$ and $y$, add $w$ to $p_{\ell}(x)$ and subtract $w$ from $p_{\ell}(y)$.

merge If we want to merge heap $P$ with heap $Q$ in such a way that the priorities in $P$ are by an offset $o$ higher than those in $Q, o$ is added to the weight of 
the dummy root edges of $P$ and in comparing the priorities of the roots of trees in $P$ to those in $Q$, the global priorities are used. Merging binomial heaps is otherwise trivial.

We note that our modifications to not increase the asymptotic complexity of the operations. Although we do not need to maintain local priorities for our algorithm, we point out that local priorities can be retained. However, in the decrease-key operation, the weight of sibling edges would need to be modified as well, and, for binomial heaps, would require $O\left(\log ^{2} n\right)$ time.

\section{B Maintaining order}

In order to maintain the left-to-right order of faces in the priority queue we refer to an order maintenance data structure DSORDER due to Dietz and Sleator 6]. DSORDER supports the following operations:

1. Insert $(X ; Y)$ : Insert a new element $Y$ immediately after element $X$ in the total order.

2. Delete $(X)$ : Remove an element $X$ from the total order.

3. $\operatorname{Order}(X ; Y)$ : Determine whether $X$ precedes $Y$ in the total order

While there are other data structures that are more efficient asymptotically [1, DSORDER is attractive for its simplicity, as it only relies on basic data structures. DSORDER is implemented as a circularly linked list that implicitly encodes the label bits to represent paths in a hypothetical $2-4$ tree and uses 2's complement arithmetic and a wrapping modulo to efficiently perform renumbering, giving:

Theorem 2. [6] The amortized time to do Insert on a list containing $n$ records is $O(\log n)$, and the amortized (and worst-case) time to do Delete or Order is $O(1)$.

DSORDER generally draws its labels from integers in $\{0, \ldots, M-1\}$, where $M$ is sufficiently larg $\AA^{3}$. Since in our algorithm every face in a newly created order is right of the faces in the previous order, we modify this range as we move left-to-right to make simple concatenation possible. I.e. if $n_{i}$ is the largest label in the order $A_{i}$, the labels for $A_{i+1}$ are drawn from $\left\{n_{i}+1, \ldots, n_{i}+M\right\}$, where $M$ is large w.r.t. the size of the graph. Then, an order $B$ created after an order $A$, can be appended to $A$ in constant time via standard linked list operations.

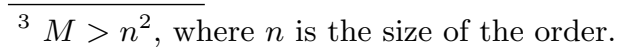

